\title{
Independent component analysis: fetal signal reconstruction from magnetocardiographic recordings
}

\author{
Silvia Comani ${ }^{a, b, *}$, Dante Mantini ${ }^{c}$, Paris Pennesi $^{c}$, Antonio Lagatta $^{b}$, \\ Giovanni Cancellieri ${ }^{\mathrm{d}}$
}
a Department of Clinical Sciences and Bio-imaging, University Foundation, Chieti University, Via dei Vestini 33, 66013 Chieti, Italy
b ITAB-Institute of Advanced Biomedical Technologies, University Foundation, Chieti University, Chieti, Italy
c Department of Informatics and Automation Engineering, Marche Polytechnic University, Ancona, Italy
d Department of Electronics, Artificial Intelligence and Telecommunications, Marche Polytechnic University, Ancona, Italy

Received 10 November 2003; accepted 10 December 2003

\author{
KEYWORDS \\ Independent component \\ analysis (ICA); \\ Fetal \\ magnetocardiography \\ (fMCG); \\ Fetal signal \\ reconstruction; \\ Data retrieval; \\ Fetal cardiac monitoring
}

\begin{abstract}
Summary Independent component analysis (ICA) was used for the processing of cardiological signals obtained by means of fetal magnetocardiography (fMCG), a technique allowing the non-invasive recording of the weak magnetic field variations associated to the electrical activity of the fetal heart. Purpose of the present work was to verify whether a computational-light ICA algorithm (FastICA), tailored to the characteristics of $f M C G$, could reconstruct reliable signals of the fetal cardiac activity during the last gestational trimester, when good electrophysiological traces are difficult to obtain although being extremely important for clinical diagnosis of severe fetal dysrhythmias. Several combinations of input recordings and output components were examined in order to assess the best configuration to successfully use FastICA. The reconstructed traces were compared with those obtained with deterministic techniques already used for this purpose, and they showed to be stable and reliable, unaffected by overlapped maternal and fetal beats and suitable for clinical applications.
\end{abstract}

(c) 2003 Elsevier Ireland Ltd. All rights reserved.

\section{Introduction}

Independent component analysis (ICA) is a signal processing technique that has demonstrated the ability of separating independent sources from mixed recorded signals when specific boundary

\footnotetext{
*Corresponding author. Tel.: +39-0871-3556901; fax: +39-0871-3556930.

E-mail address: comani@itab.unich.it (S. Comani).
}

conditions are satisfied. ICA represents a multidimensional random vector as a linear combination of random variables (independent components) that are as independent as possible. Several efficient algorithms utilizing higher order statistics have been improved and can be used to solve the ICA problem, which consists in separating the independent sources of the mixed signals [1].

ICA has recently received attention because of its potential applications in several fields, as 
demonstrated in studies on specific problems dealing with speech recognition systems, telecommunications and medical signal processing [2-5]. ICA techniques can also be applied to non-conventional problems, because the basic assumption of the ICA problem, i.e. the independence of the variables, is realistic in many situations, hence permitting a completely blind source signals separation, or independent components retrieval, starting from given mixed input signals $[6,7]$.

The successful applications of ICA in several fields where data analysis, source separation or feature retrieval were required $[8,9]$, had encouraged its use also in biological signal processing, which are in general more complex to analyze than non-biological systems [10-14]. An intriguing application of ICA regards the processing of cardiological signals. The data we used as input were acquired by means of magnetocardiography (MCG), a technique that allows the non-invasive detection of the extremely low magnetic field variations associated to the spontaneous electrical activity of the human heart [15]. A particular application of MCG is fetal magnetocardiography (fMCG), which records the magnetic field variations occurring over the abdomen of a pregnant woman; the data obtained with PMCG contain a combination of signals that are due to the electrical activity of both the fetal and the maternal hearts, since both are included in the same space volume, i.e. the maternal abdomen.

The ICA prerequisites for independent sources and for mixed recorded traces are satisfied with fMCG data; in fact, pregnancy is a unique physiological condition in which a healthy human being has two distinct beating hearts, which, from the mathematical point of view, are the independent signal sources.

The ICA problem is, however, rather complex in the case of fMCG data; one reason for this complexity is that fetal and maternal cardiac signals are both quasi-periodic and have a similar waveform, which is the electromagnetic expression of the atrial and ventricular depolarization and repolarization processes taking place during the cardiac cycle. Notwithstanding their similar shape, the fetal and maternal cardiac signals are characterized by different values of frequency (the fetal heart rate is higher than the maternal one) and intensity (the maternal heart is bigger than the fetal one and therefore produces a stronger signal). As a consequence, some weak fetal beats may be hidden behind the more intense maternal beats.

As a further complication, no heart rate is ever perfectly constant; this condition implies that the source signals to be retrieved are not strictly periodic; on the contrary, they are characterized by a variability that, in case of cardiac disease, may also drastically increase.

Finally, the weakness of the fetal signals $\left(\approx 10^{-12} \mathrm{~T}\right)$, the amplitude of which is sometimes comparable to the magnetic background noise, further complicates the process of recognition and extraction of the fetal components from the recorded signals.

Despite these problems, a correct evaluation of the fetal cardiac electrical activity is essential to neonatologists and gynecologists for antenatal and intrapartum surveillance.

It is evident that an early detection of cardiac disease, such as fetal dysrhythmias, is of great importance to plan a correct therapeutic intervention; in fact, fetal dysrhythmias may trigger a reentrant tachyarrhythmia that might lead to cardiac insufficiency or even to fetal death [16]. Therefore, techniques able not only to monitor the fetal heart rate but also to provide accurate trace morphology are required.

Among the non-invasive methods available for the antenatal monitoring of the fetal cardiac activity in the clinical practice, cardiotocogram (CTG) and fetal electrocardiogram (fECG) can be used, but both present severe disadvantages. CTG, based on pressure changes at the level of the maternal abdomen, generally provides accurate information only about the fetal heart rate (FHR) [17]. On the other hand, fECG, obtained with electrodes positioned on the maternal abdomen, produces weak signals where the shape of the fetal QRS complex can be recognized, but other intra-cardiac intervals, useful to characterize fetal arrhythmias, can rarely be appreciated. Moreover, the intensity of fECG signals, normally dependent on several uncontrolled factors such as the gestational age, the fetus orientation inside the maternal abdomen or the amount of maternal electrical contamination in the recordings, drastically decreases during the last trimester of gestation because of the insulating properties of the vernix caseosa, an ensemble of desquamative epithelial cells surrounding the fetus and having a resistance factor $10^{6}$ times higher than the nearby tissue $[17,18]$.

As a consequence, m-mode and Doppler ultrasonography is, in general, the method of choice in the clinical setting, since it allows detecting the mechanical events that follow the electrical phases of the cardiac cycle. Fetal bradycardia, supraventricular tachycardia and extra-systole are therefore diagnosed by means of ultrasound, but the timing is not precise and the intrinsic electrical nature of these events cannot be described $[16,19]$.

Recent studies have reported evidence of the usefulness of PMCG in the detection of fetal 
arrhythmias and in the evaluation of important parameters for its classification [20,21] since the magnetic field variations are almost unaffected by the vernix caseosa [22]. Therefore, fMCG seems an appropriate technique for the monitoring of the fetal cardiac activity during the prenatal period, but it requires to be supported by reliable techniques for the reconstruction of accurate fetal traces from the intertwined maternal and fetal recordings [7,23-27].

A computational light Fixed Point ICA algorithm (FastICA) was chosen and tailored to our MCG system characteristics in order to reconstruct trustworthy fetal cardiac signals.

\section{Materials and methods}

\subsection{MCG system characteristics}

MCG signals, characterized by very weak amplitudes that can hardly be distinguished from noise, require the use of tools that must be sensible to the extremely low variations of the magnetic field associated to bioelectrical activity. The rapid technological progress of MCG instrumentation is due to the combination of two factors: (1) the development of Superconducting Quantum Interference Devices (SQUIDs) that provide the required performance by converting, with great sensitivity, a variable induction flow through a superconducting loop into a measurable electric tension; (2) the availability of multi-channel systems that allow the simultaneous recording of the bio-magnetic field variations from several positions over the chest or abdomen [28-32]. The reduction of environmental noise in the recorded signals can be either obtained by applying a strong physical shielding or by using differential measures.

The MCG system used for this study is a flat 77-channel system operating in a shielded room (Fig. 1). Each channel consists of a low-temperature dc-SQUID integrated magnetometer with the sensitivity of approximately $5 \mathrm{fT} / \sqrt{\mathrm{Hz}}$ [33]. The 77 magnetometers are kept at liquid helium temperature inside a cylindrical cryostat; they are grouped in two sets: one set is composed of 55 measuring magnetometers, which are arranged at fixed distances $(32 \mathrm{~mm})$ in a honeycomb grid of $230 \mathrm{~mm}$ diameter and are ideally lying on a sensing plane close to the cryostat bottom; the other set includes the remaining magnetometers, which are distributed on higher planes for residual noise reduction (Fig. 2). The planar geometry of this system is particularly convenient for measurements over the chest and abdomen.

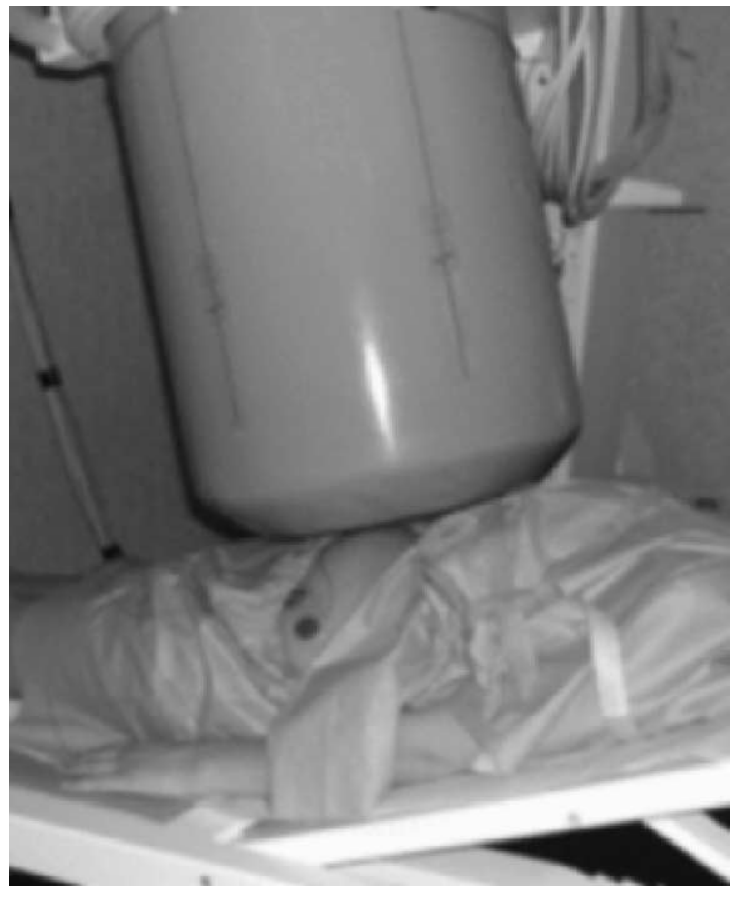

Fig. 1 View of the multi-channel MCG system for a Fetal MCG recording session. The system works inside a shielded room and is positioned over the maternal abdomen; the couch designed for maximal maternal comfort is also visible.

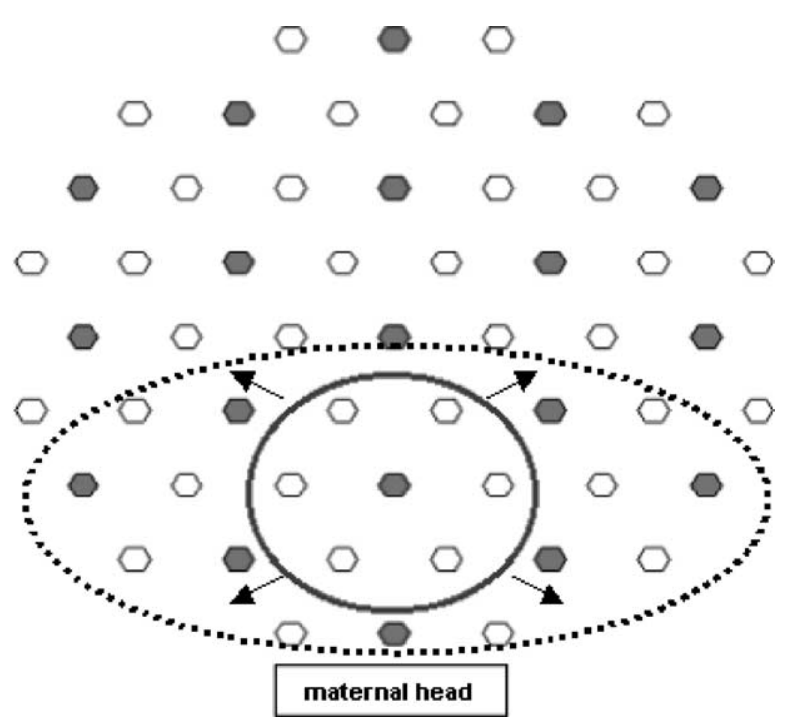

Fig. 2 Arrangement of the 55 sensing magnetometers placed on the sensing plane. The gray hexagons indicate the positions occupied by the magnetometers placed on higher planes and used for residual noise correction. The full-line circle indicates a 7-channel cluster (construction cluster), which can be enlarged up to a 22-channel cluster, as indicated by the dashed line. The position of the maternal head relatively to the acquisition magnetometers is also indicated. 


\subsection{MCG signal acquisitions}

After written informed consent, twenty-three pregnant women with a singleton pregnancy, age between 23 and 40 years, participated in the study, approved by the Ethics Committee of our University. All volunteers presented no cardiac disease and a normal pregnancy. Two to four $\mathrm{AMCG}$ acquisition sessions were performed for each volunteer at several gestational ages from the 22nd week until delivery.

A morphological and functional fetal heart evaluation by means of $\mathrm{m}-2 \mathrm{D}$ pulsed color Doppler echocardiogram was performed prior to fMCG recording for reference; it also provided information about the projection of the fetal heart on the maternal abdomen to allow a correct positioning of the MCG system. fMCG signals were acquired for about $5 \mathrm{~min}$ with a sampling rate of $10 \mathrm{kHz}$, and converted into digital form with a resolution of 8 bits. Acquired data were transferred from the acquisition system to the post-processing station via a high-speed optical connection. Digital band-pass data filtering was performed for a bandwidth extended from 0.016 to $250.0 \mathrm{~Hz}$. Data were decimated by a factor of 10 in order to reduce the total amount of data to be handled. Fifty-five raw data files with a sampling frequency of $1 \mathrm{kHz}$ were finally available for analysis.

\subsection{ICA and fMCG: is it possible?}

As regards ICA fundamental hypotheses, the assumption of independence of the signal sources in fMCG is satisfied, since the maternal and fetal hearts are surely physically distinct. Furthermore, the maternal and fetal magnetocardiographic signals, although being similar, differ from each other for the values assumed by the signal period and intensity. As a consequence, ICA can theoretically provide a relationship between a vector containing the acquired raw signals and another vector containing the separated maternal and fetal traces.

\subsection{Independent component analysis (ICA)}

The basic ICA model $[1,6,7,34]$ can be shortly described as follows. Denote the $n$-dimensional data vector by $x(t)=\left[x_{1}(t), \ldots, x_{n}(t)\right]^{\top}$ and the $m$-dimensional vector of independent components $s_{i}(t)$ by $s(t)=\left[s_{1}(t), \ldots, s_{m}(t)\right]^{\top}$. It is assumed that $s_{i}(t)$ are zero mean, and at most one of them has a Gaussian distribution. The ICA linear expansion

$x(t)=A s(t)$ is fitted to the data vector $x(t)$, where $A=$ $\left[a_{1}, \ldots, a_{m}\right]$ is a constant full-rank $[n \times m]$ matrix, named mixing matrix. In general, the number of expected independent components has to be at most equal to the number of mixtures, i.e. to the number of the components of the input data vector. Usually the component $s_{i}(t)$ with Gaussian probability distribution corresponds to background noise.

The vector containing the observed signals $x(t)$ is linearly transformed into another vector $x^{\prime}(t)=M x(t)$ (whitening); afterwards, we can state that $E\left\{x^{\prime} x^{\top}\right\}=I$. This transformation can be accomplished by means of Principal Component Analysis.

The independent components, or source signals, can be found by determining a $[m \times n]$ matrix $W$, named de-mixing matrix, such as

$y(t)=W x^{\prime}(t)$

The $m$-dimensional vector $y(t)$ becomes a good estimate of the source vector $s(t)$.

The best $y(t)$ vector can be found by minimizing the following functional

$I\left(p_{y}\right)=\int p_{y}(u) \log \frac{p_{y}(u)}{\prod_{i=1}^{N} p_{y i}(u)} \mathrm{d} u$

$I\left(p_{y}\right)$ depends on $W$ through Eq. (2); $p_{y}$ is the probability distribution function associated to a random vector $y, p_{y i}$ is the probability distribution function associated to the $i$ th component of vector $y$. The functional $I\left(p_{y}\right)$ expresses the average mutual information and derives from the Kullback divergence, which measures the distance between two probability distribution functions. In order to approach the ideal case in which $p_{y}=\prod_{i=1}^{N} p_{y i}$, the functional $I\left(p_{y}\right)$ has to be minimized.

\subsection{FastICA algorithm}

Most suggested solutions to the ICA problem $[35,36]$ use the kurtosis (fourth-order cumulant) of the signals, defined for a zero-mean random variable $v$ as

$\operatorname{kurt}(v)=E\left\{v^{4}\right\}-3\left(E\left\{v^{2}\right\}\right)^{2}$

For a Gaussian random variable, kurtosis is null; for densities peaked at zero, it is positive, and for flatter densities, it is negative. It is worth noting that for two independent random variables $v_{1}$ and $v_{2}$ and for a scalar $\alpha$, the following properties have to be considered:

$$
\begin{aligned}
& \operatorname{kurt}\left(v_{1}+v_{2}\right)=\operatorname{kurt}\left(v_{1}\right)+\operatorname{kurt}\left(v_{2}\right), \\
& \operatorname{kurt}\left(\alpha v_{1}\right)=\alpha^{4} \operatorname{kurt}\left(v_{1}\right)
\end{aligned}
$$

At this point, it is necessary to find a linear combination of observed components that has maximal 
or minimal kurtosis. Using the orthogonal matrix $B$, we can define

$$
\begin{aligned}
\operatorname{kurt}\left(w^{\top} x^{\prime}\right) & =\operatorname{kurt}\left(w^{\top} B s\right) \\
& =\operatorname{kurt}\left(z^{\top} s\right)=\sum_{i=1}^{n} z_{i}^{4} \operatorname{kurt}\left(s_{i}\right)
\end{aligned}
$$

Under the assumption that ||$w\|=\| z \|=1$, a discrete number of local maxima and minima can be found in the function given by (6). Consequently, the columns of the mixing matrix A can be calculated as solution for $w$ by minimizing or by maximizing the kurtosis in (6) under the given constraint, and the linear combination $w^{\top} x$ will be one of the required independent components:

$w^{\top} x^{\prime}=\left(b_{i}\right)^{\top} x^{\prime}=s_{i}$

In order to maximize or minimize $\operatorname{kurt}\left(w^{\top} x^{\prime}\right)$, some neural algorithms based on natural gradient can be used [1]. Considering that input signals have been pre-whitened, it will be

$$
\begin{aligned}
\operatorname{kurt}\left(w^{\top} x^{\prime}\right) & =E\left\{\left(w^{\top} x^{\prime}\right)^{4}\right\}-3\left[E\left\{\left(w^{\top} x^{\prime}\right)^{2}\right\}\right]^{2} \\
& =E\left\{\left(w^{\top} x^{\prime}\right)^{4}\right\}-3\|w\|^{4}
\end{aligned}
$$

Therefore, the function to be minimized can be simplified in the following way:

$$
J(w)=E\left\{\left(w^{T} x^{\prime}\right)^{4}\right\}-3\|w\|^{4}+F\left(\|w\|^{2}\right)
$$

where $F$ is a penalty term due to the constraint $\|w\|=1$. In the case of kurtosis minimization and/or maximization, the learning rule will have the following form:

$$
\begin{aligned}
w(t+1)= & w(t) \pm \mu(t)\left[x^{\prime}(t)\left(w(t)^{T} x^{\prime}(t)\right)^{3}\right. \\
& \left.-3\|w(t)\|^{2} w(t)+f\left(\|w(t)\|^{2}\right) w(t)\right]
\end{aligned}
$$

where $x^{\prime}(t)$ is the sequence of observed signals, $\mu(t)$ is the learning rate sequence, and $f$ is the derivative of $F / 2$.

The learning rule, described in (10), will stop at a fixed point for which the expected change is zero. This condition is given by:

$$
\begin{aligned}
\frac{\mathrm{d}(J(w))}{\mathrm{d} w} & =E\left\{x^{\prime}\left(w^{\top} x^{\prime}\right)^{3}\right\}-3\|w\|^{2} w+f\left(\|w\|^{2}\right) w \\
& =0
\end{aligned}
$$

In order to solve the ICA problem concerning fetal magnetocardiographic data, we preferred to use FastICA algorithm, also called Fixed-point iteration ICA, because it is an algorithm particularly light from the point of view of the required computational effort.
FastlCA version 2.1 was implemented by its inventors using MATLAB 5.x. Such program, freely acquired from Internet for research purpose [37], was installed in our laboratory on an Intel P3 PC (256 MB RAM), with Windows O.S.

The FastICA algorithm $[36,38]$ derives from the theory described from Eqs. (4)-(11). It consists of three steps:

1. an initial vector $w(0)$ is chosen randomly and normalized in order to have a unit norm.

2. the subsequent estimate of $w$ is computed by using the fixed-point iteration rule

$$
w(k)=E\left\{x^{\prime}\left(w(k-1)^{\top} x^{\prime}\right)^{3}\right\}-3 w(k-1)
$$

where $w(k)$ is normalized by dividing it by its norm.

3. step no. 2 is repeated until $\left|w^{\top}(k) w(k-1)\right|$ is estimated to be sufficiently close to unity.

This procedure is used to estimate one ICA basis vector. If necessary, other ICA basis vectors can be estimated by sequentially projecting a new starting basis vector $w(0)$ onto the subspace that is orthogonal to the subspace spanned by the previous ICA basis vectors, and after that by repeating the described procedure from step 1 to step 3 .

A remarkable property of the FastICA algorithm is its cubic convergence speed. In fact, it permits to obtain a high accuracy with a very small number of iterations [38]. In general, the accuracy depends on the input data quality, in terms of temporal resolution and signal-to-noise ratio.

\subsection{FastICA tailoring to $\mathrm{AMCG}$ signals}

The tailoring of FastICA to $\mathrm{MCG}$ recordings and best fetal trace reconstruction can be distinguished in three subsequent phases.

\subsubsection{Phase 1: fMCG data pre-processing} Before running the FastICA algorithm, some pre-processing was performed on raw fMCG data in order to provide FastICA with suitable data that might be less affected by residual noise:

1. raw traces were grouped into clusters each having $N_{1}$ traces $\left(N_{1} \leq 55\right)$; clustering was made following the system geometry and/or other criteria regarding the spatial distribution of channels or the intensity of the signals;

2. simultaneous trace intervals, sufficiently long for a FastICA run and presenting very low baseline shifts, were chosen for the traces of the same cluster;

3. selected trace intervals were filtered by means of a FIR filter, with band $0.4-150.0 \mathrm{~Hz}$ [39]; 
4. trace intervals were decimated by a factor 3 in order to reduce the total amount of data to be handled without modifying the signal spectrum, but simultaneously reducing noise; in fact, no relevant spectral component was present above $150 \mathrm{~Hz}$ [39];

5. the number $N_{0}$ of required output independent components was chosen $\left(N_{O} \leq N_{1}\right)$.

\subsubsection{Phase 2: FastICA iterations}

After data pre-processing, FastICA iterations were performed using the algorithm described in 2.5. The required output was a set of $N_{0}$ traces, which will always be displayed in random order. The intensity of the extracted components was given in arbitrary units, and the polarity of the signals might be inverted with respect to the input data. Generally, a FastICA output includes:

- fetal signal components, which present a higher heart-rate;

- maternal signal components, which present a lower heart-rate;

- white noise.

Usually, one to three components can be ascribed to the signal originating from the fetal heart.

\subsubsection{Phase 3: Fetal trace reconstruction}

The elements of the de-mixing matrix $\mathrm{W}$ contain the information necessary to reproduce the correct amplitudes in the output FastICA components, on the basis of (2). Among the $y(t)$ components, the ones related to the fetal cardiac activity can be selected on account of the heart rate and then combined to reproduce the final fetal signal. Since the components have random polarity, the components with inverse polarity can be retrieved coherently with the original data by multiplying them by a factor -1 ; finally the fetal components are summed to reconstruct the fetal signal. In this way the correct intensity scales are restored; moreover, the appropriate time scale is obtained by interpolation.

As evident from the description of the overall procedure, each input cluster can provide only one reconstructed fetal trace that can be eventually filtered and/or smoothed in order to obtain signals characterized by more easily recognizable waveforms. Fig. 3 provides a diagram that summarizes the three phases of the fetal trace reconstruction.

\subsection{Validation of the FastICA effectiveness}

Our analysis aimed at verifying the suitability of FastICA to reconstruct cardiac fetal traces, but it also regarded the choice of the best configuration

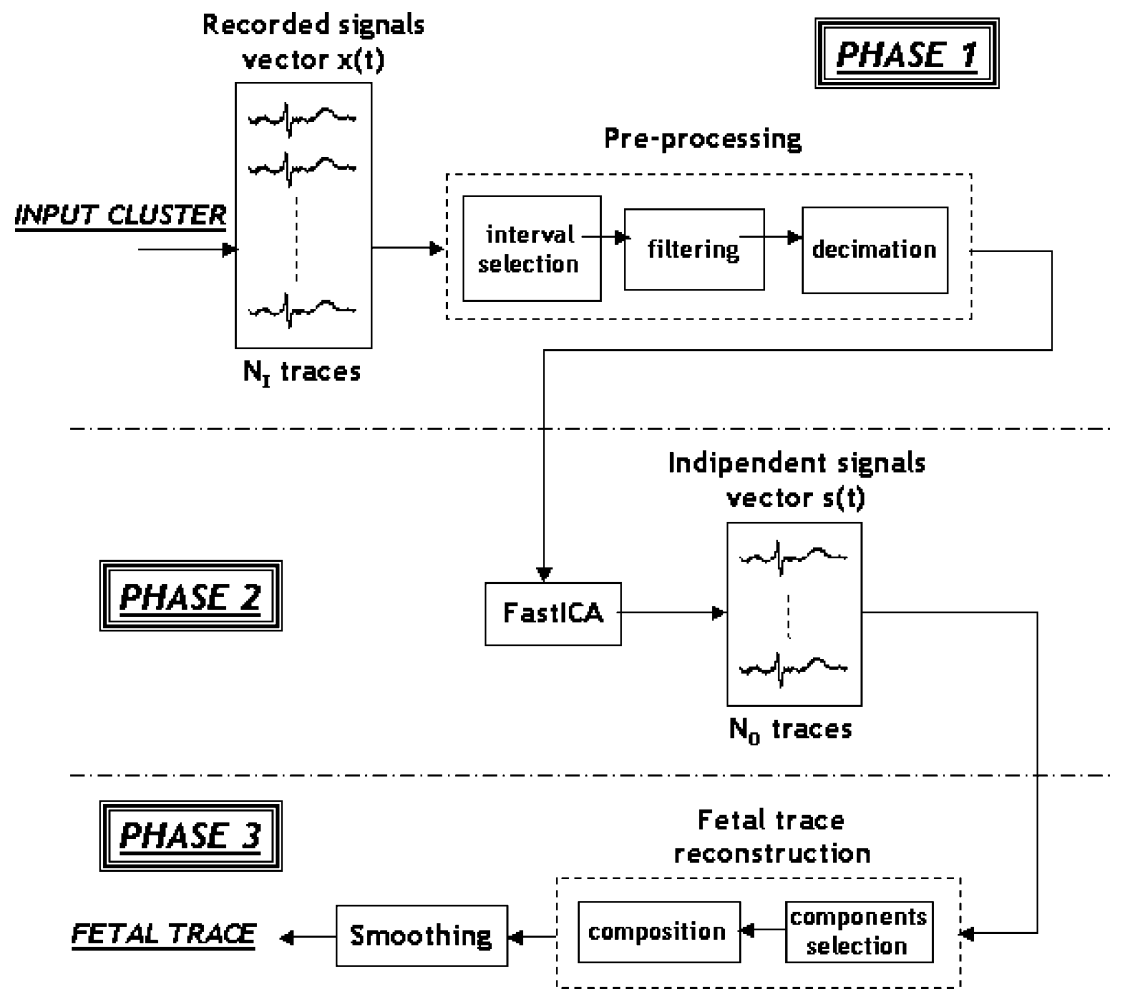

Fig. 3 Diagram summarizing the various steps in the reconstruction of a fetal signal. The three processing phases described in Section 2.6 are summarized and sequentially shown. 
for the input cluster and for the number of independent components necessary to reconstruct a reliable fetal signal. Several attempts have been made to find the optimal recordings number and configuration for the input cluster in combination with an ideal number of required output traces.

Since the magnetocardiographic technique allows the simultaneous recording of the cardiac signal from different positions, hence permitting to follow the evolution of 2-D magnetic maps during the cardiac cycle, it would have been useful to maintain a fetal mapping also after fetal signal reconstruction. In order to achieve this objective, we tried to restore the fetal signals using small input clusters, given that the ICA procedure permitted to retrieve only one trace from a given number $N_{1}$ of input recordings. In case of success, we would have obtained a reconstruction of the fetal cardiac signal not only in the time, but also in the space.

By following the honeycomb geometry of the MCG system, each input cluster was then composed by the seven traces recorded by the seven channels belonging to the same construction cluster (see Fig. 2). In combination with this input setting, an output of two to six components was sequentially required, in agreement with the FastICA constraint on the allowed number of output traces, which must be at most equal to the number of input traces. In order to evaluate the lowest number of input traces necessary to obtain a stable output, larger clusters with an increased number of input channels were also used. With reference to the construction geometry of the acquisition system, the 7-channel cluster was enlarged with the addition of adjacent channels, as shown in Fig. 2. Input clusters of 12, 16 and 22 traces, respectively, were finally utilized.

The reliability of the FastICA results obtained with the various channel configurations was estimated on the basis of the stability and quality of the reconstructed traces.

The stability of the FastICA output was related to the reproducibility of the same results with repetitive FastICA runs.

The quality of the fetal signals was estimated by comparing the traces with those obtained, from the same data sets, by means of another method using a deterministic approach working in the time domain and eliminating the averaged maternal beat from the fMCG recordings. The signals intensities and periods were compared with the ranges of normality for this kind of signals [26].

A rate of success (RRS) in the reconstruction of the fetal signals for different gestational epochs was calculated. It was defined as the number of reconstructed fetal signals over the number of recorded sets and regarded signals in which at least the ventricular depolarization wave could be clearly identified. The useful rate of success (URS) was defined in function of the possible use of such fetal signals for more detailed clinical analysis; it was defined as the number of good fetal signals, allowing the recognition of atrial and ventricular waves, over the number of reconstructed signals. An overall rate of success (ORS) was also defined as the number of good fetal signals over the number of recorded sets. The values obtained for these indices were compared with those available in the literature [40].

\section{Results}

A total of 61 sets of fMCG recordings, spanned from the 22nd to the 37th gestational week, were available for analysis. All volunteers had normal findings at M-2D pulsed color Doppler ultrasonography. The FastICA appropriateness to reconstruct fetal signals depended on the reliability of the results, which, in turn, could be estimated from the stability and quality of the recovered traces.

An important point of our analysis regarded the choice of the best configuration of input recordings and number of required independent components; it was evident that trace stability depended on this choice. All combinations described in Section 2.7, were tried with all recordings sets; stable and repeatable output components could only be obtained with the use of an input cluster of 22 recordings and an output set of six components. The use of smaller input clusters and/or output component sets (desirable for mapping purposes) could occasionally bring to the separation of the fetal and maternal signals, but the output was unstable and the waveform of the independent components rather unpredictable: from one FastICA iteration to the next different results and unrecognizable cardiac signals could be obtained.

Figs. 4 and 5 show an example in which a 7-channel input cluster together with two output components succeeded in separating the maternal and fetal traces, although this output was not stable. Cases like this one were rare and usually referred to recordings performed during the late gestational weeks, when, in general, stronger signals are available. The data shown in these pictures refer to a woman at her 35th week of gestation.

Similar results were obtained with the use of a 7-channel input cluster and six output components, as shown in Fig. 6. In general, the increased number of required components allowed retrieving more information on the fetal cardiac signal; nonetheless, the output sets obtained with this combination of clusters continued to be unstable. The data shown 

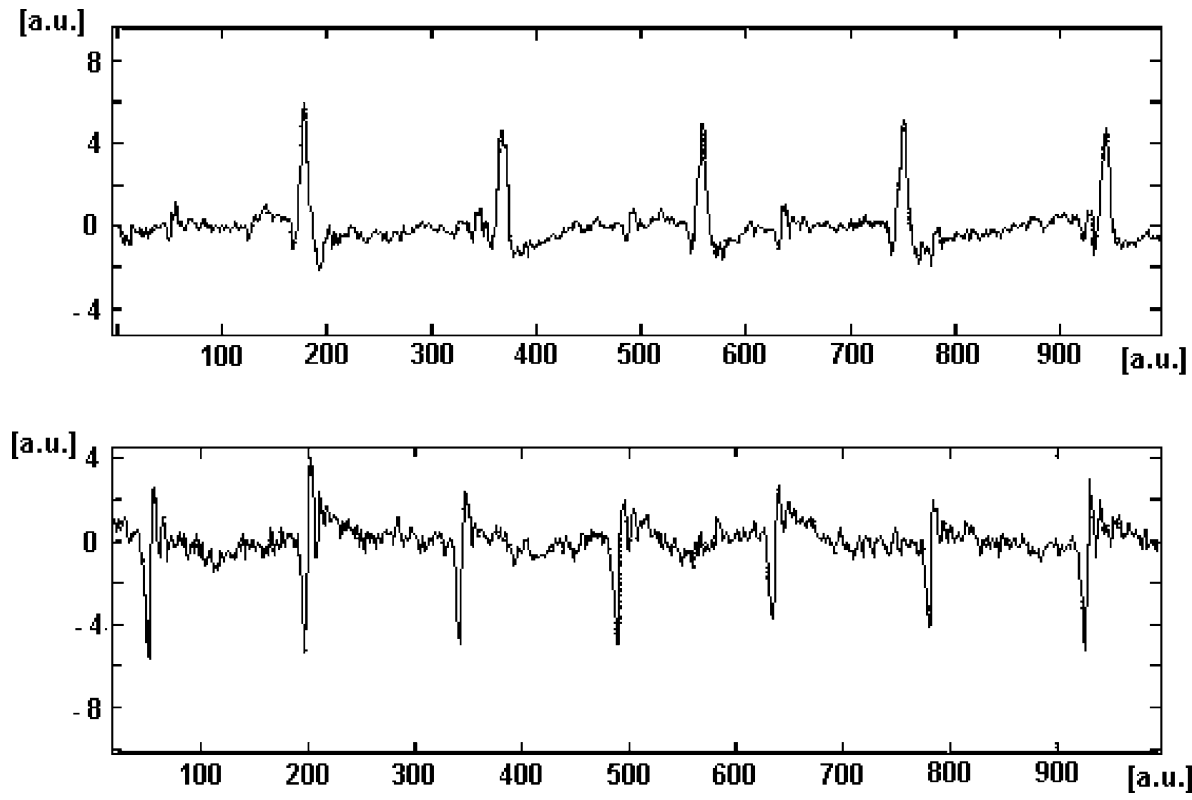

Fig. 4 Example of maternal and fetal signals reconstructed with the use of a 7-channel input cluster. Only two output components were required. The upper component is the maternal trace, and the lower component is the fetal trace, as it can be stated from the value of the heart rate. The order of appearance of the output components is random. Arbitrary units are used for the $X$-axis (time) and the $Y$-axis (amplitude).
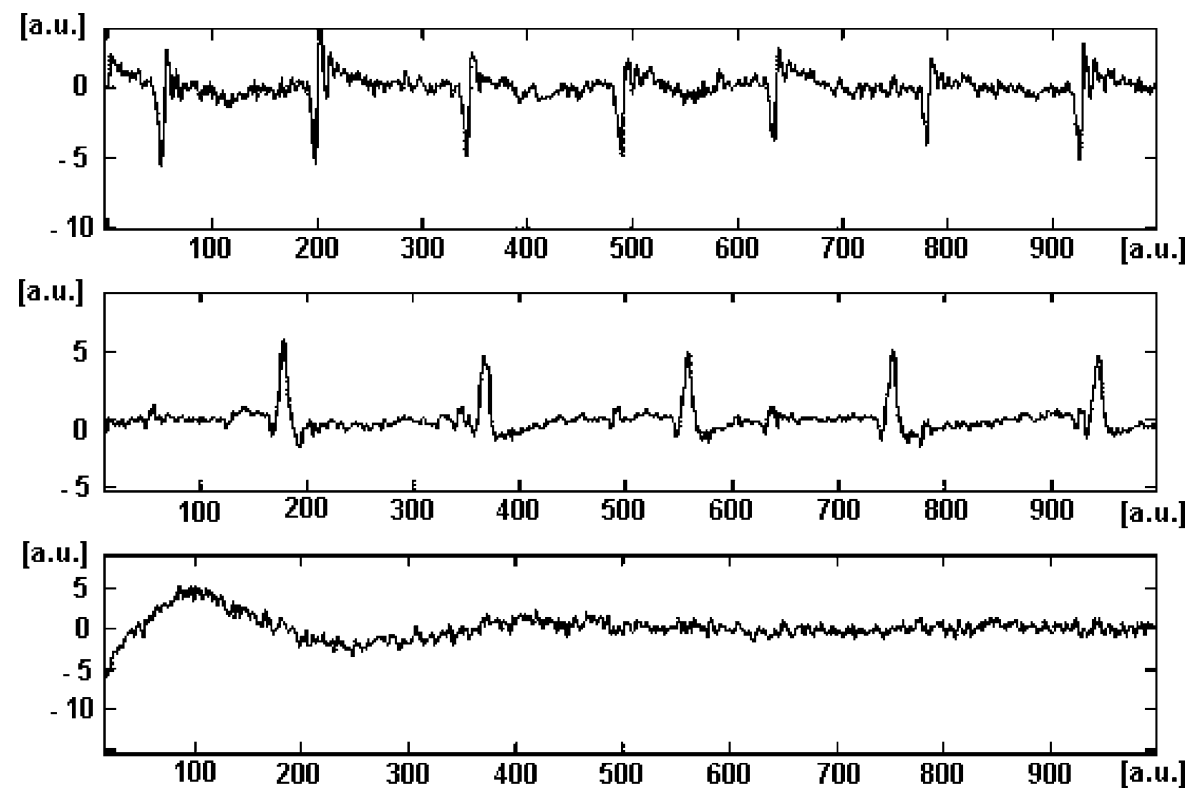

Fig. 5 Example of maternal and fetal signals reconstructed with the use of a 7-channel input cluster. The number of required output components was set equal to three. The upper component is the fetal trace; the middle component is the maternal trace, and the lower component is background noise. The order of appearance of the output components is random. Arbitrary units are used for the $X$-axis (time) and the $Y$-axis (amplitude).

in this picture refer to the same woman as in Figs. 4 and 5.

The results attained with larger input clusters (12 and 16 recordings) and six output components were, in general, more consistent, although some residual instability and the occurrence of mixed ma- ternal and fetal traces were observed. These features were observed more frequently for recording sets related to earlier gestational periods, i.e. below the 32nd gestational week. Figs. 7 and 8 show the findings for the same volunteer as in the other plots. 


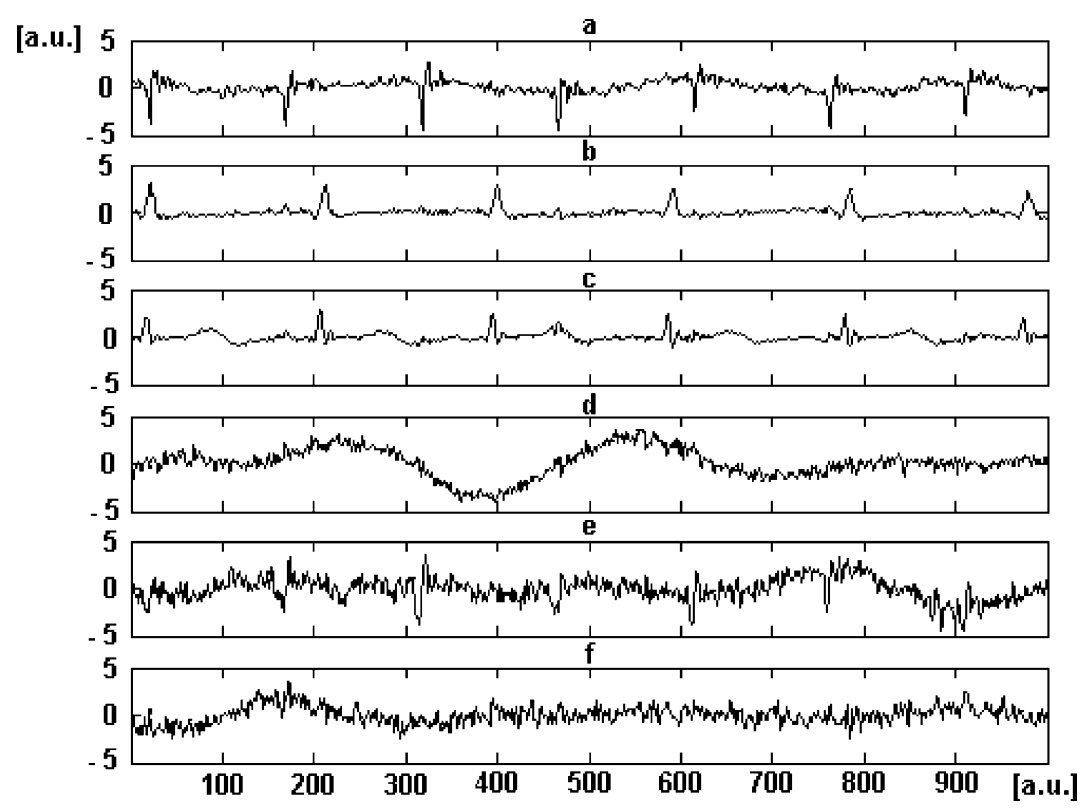

Fig. 6 Example of maternal and fetal components extracted with the use of a 7-channel input cluster and six output components. Two fetal components ('a' and 'e') can be recognized, as well as two maternal components (' $b$ ' and ' $c$ '); two traces ('d' and ' $f$ ') are noise components. The order of appearance of the output components is random. Arbitrary units are used for the $X$-axis (time) and the $Y$-axis (amplitude).

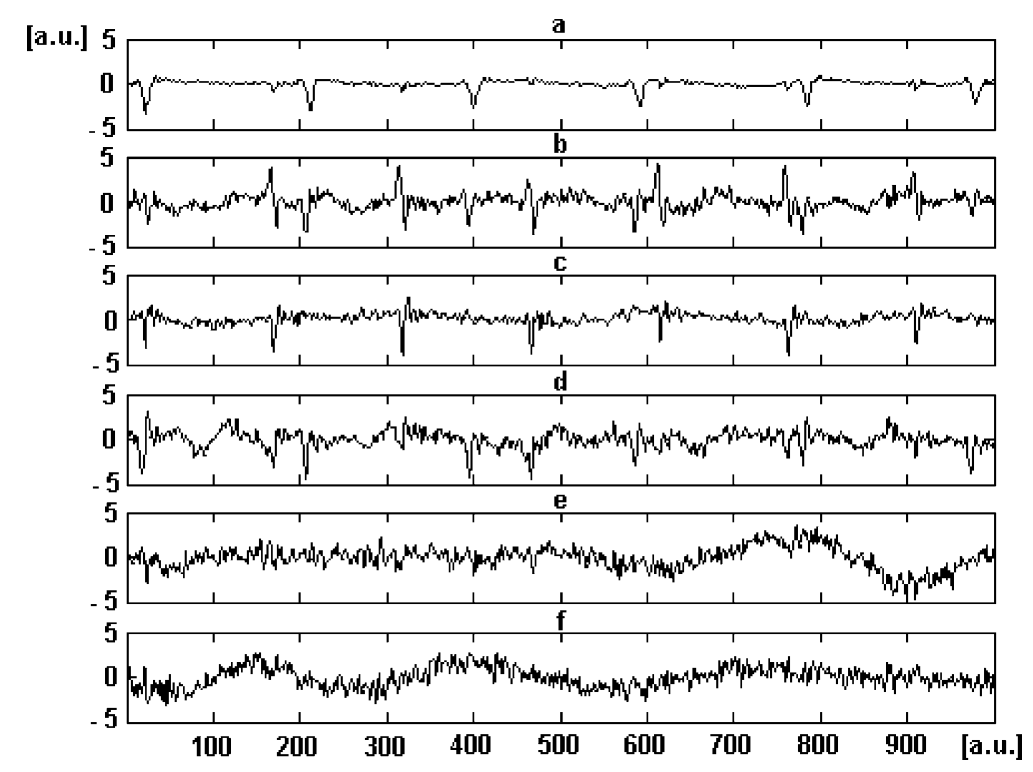

Fig. 7 Example of maternal and fetal components extracted with a 12-channel input cluster and six output components. One fetal component can be observed ('c'), as well as one maternal component (trace ' $a$ '); mixed maternal and fetal components ('b' and 'd') and two noise components ('e' and ' $f$ ') are also present. The order of appearance of the output components is random. Arbitrary units were used for the $X$-axis (time) and the $Y$-axis (amplitude).

As anticipated, the best results were achieved with the use of large input clusters of 22 recordings and six independent components as output; Fig. 9 shows the components extracted for the same data set as in the other pictures. It was shown that further increasing the number of the output traces did not add significant information. This combina- tion of clusters produced a stable output, which means that several FastICA iterations always provided the same independent components, although their order, which is random, continued to be unpredictable.

The fetal components could then be selected on account of the heart rate and subsequently com- 


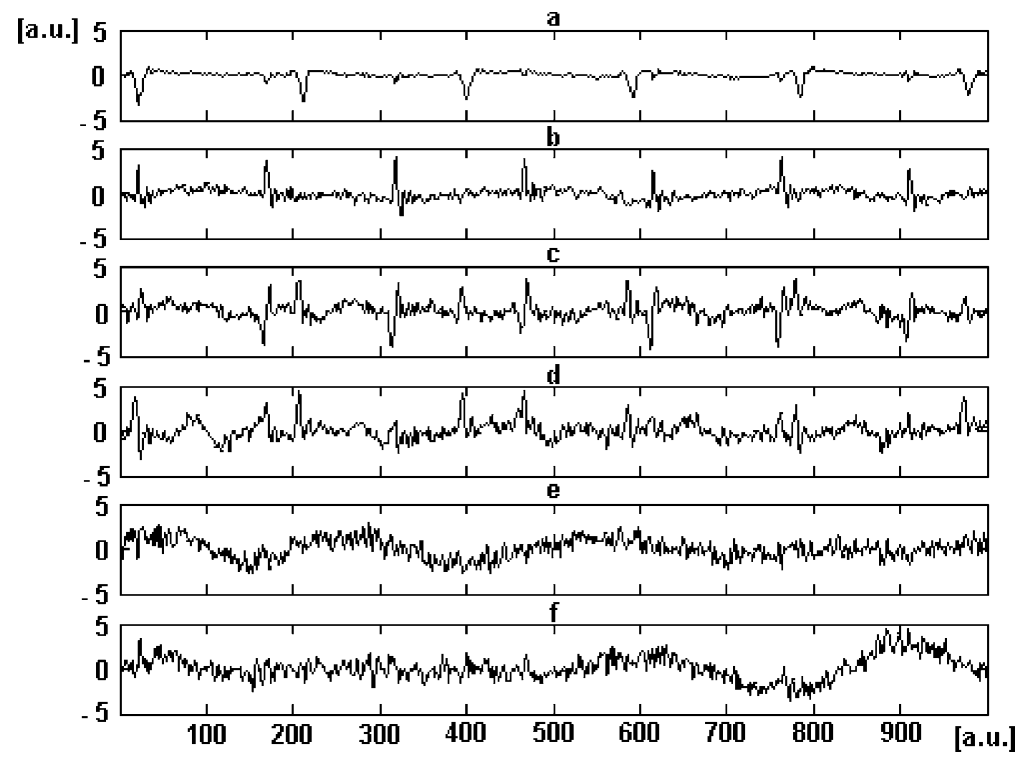

Fig. 8 Example of maternal and fetal components extracted using a 16-channel input cluster and six output components. One fetal component can be observed ('b'), as well as one maternal component ('a'); mixed maternal and fetal components ('c' and 'd') and two noise components (' $e$ ' and ' $f$ ') are also present. The order of appearance of the output components is random. Arbitrary units were used for the $X$-axis (time) and the $Y$-axis (amplitude).

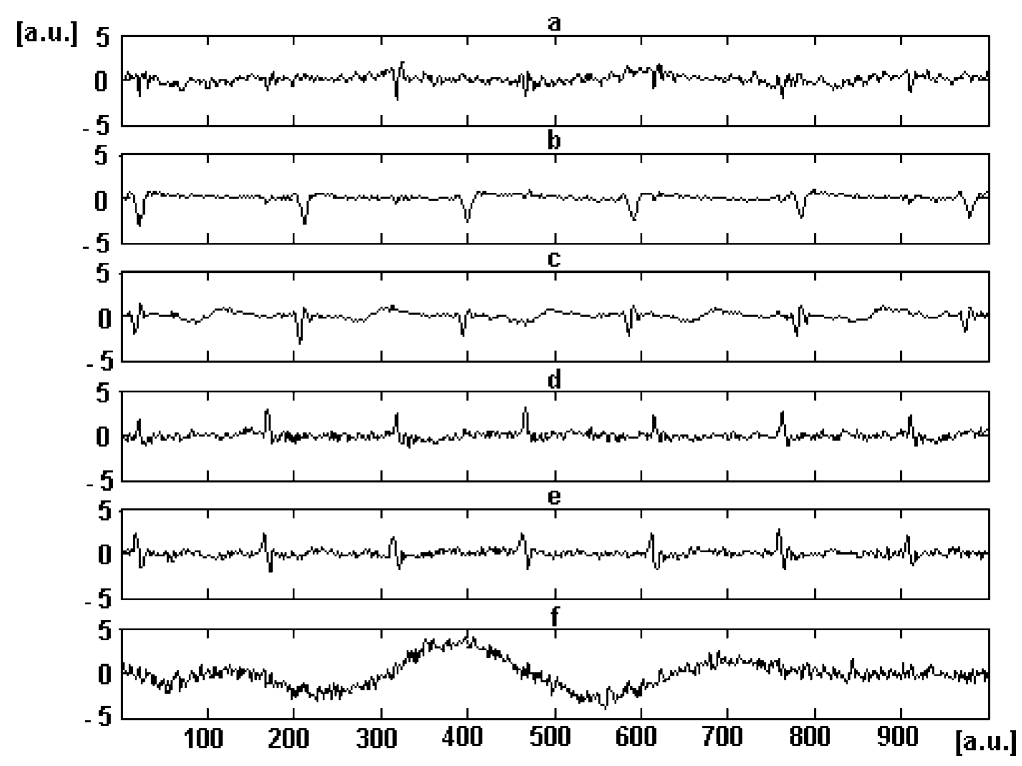

Fig. 9 Example of maternal and fetal components extracted from a 22-channel input cluster. The six output components were stable. It is possible to recognize three fetal components ('a', 'd', 'e'), two maternal components ('b' and ' $c$ ') and one noise component (' $f$ '). The order of appearance of the output components is random. Arbitrary units were used for the $X$-axis (time) and the $Y$-axis (amplitude).

bined as described in Section 2.6.3 to reproduce the final fetal signal. The correct amplitude and temporal scales were also restored (Fig. 10).

In Fig. 11 is given an example of the effect of filtering and smoothing procedures: the reconstructed fetal trace is treated in order to have a clearer definition of the cardiac waves for post-processing purposes.
The stability observed in the case shown in Figs. 10 and 11 was also experienced in all analyzed data sets from which a fetal signal could be retrieved. There were data sets from which no fetal signal could be reconstructed, but in those cases the deterministic method failed as well. On the contrary, it should be underlined that in some cases, mainly referring to early gestational periods, 


\section{maternal signal}

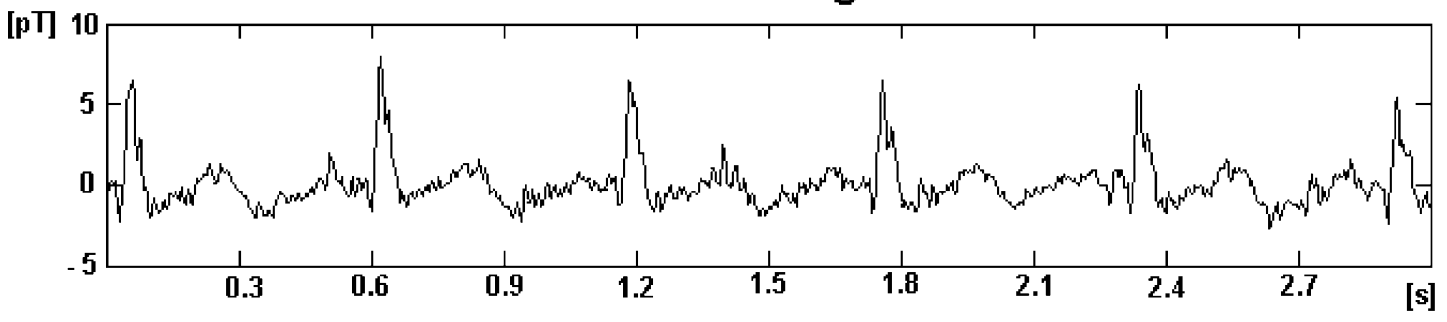

fetal signal

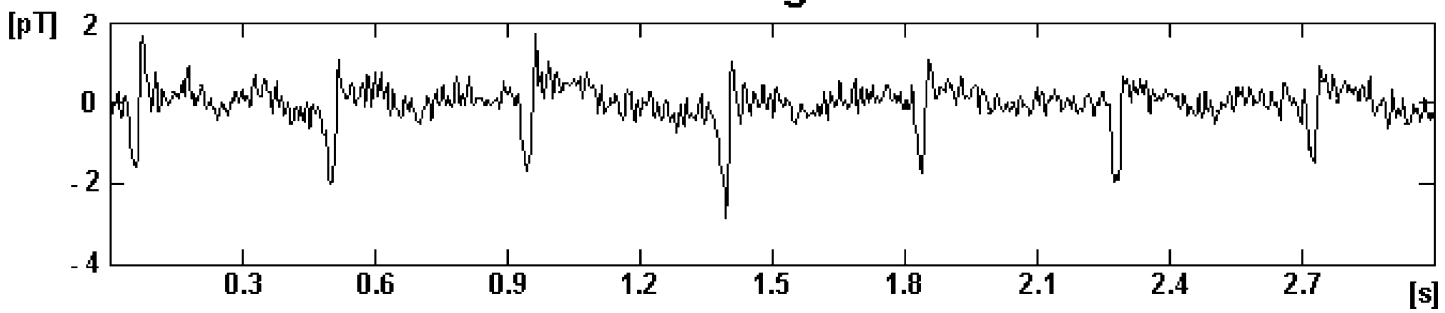

Fig. 10 Example of reconstructed maternal and fetal signals. The 6 output components obtained from a 22-channel input cluster were used. The correct amplitude (pT) and time (s) scales have been reconstructed.

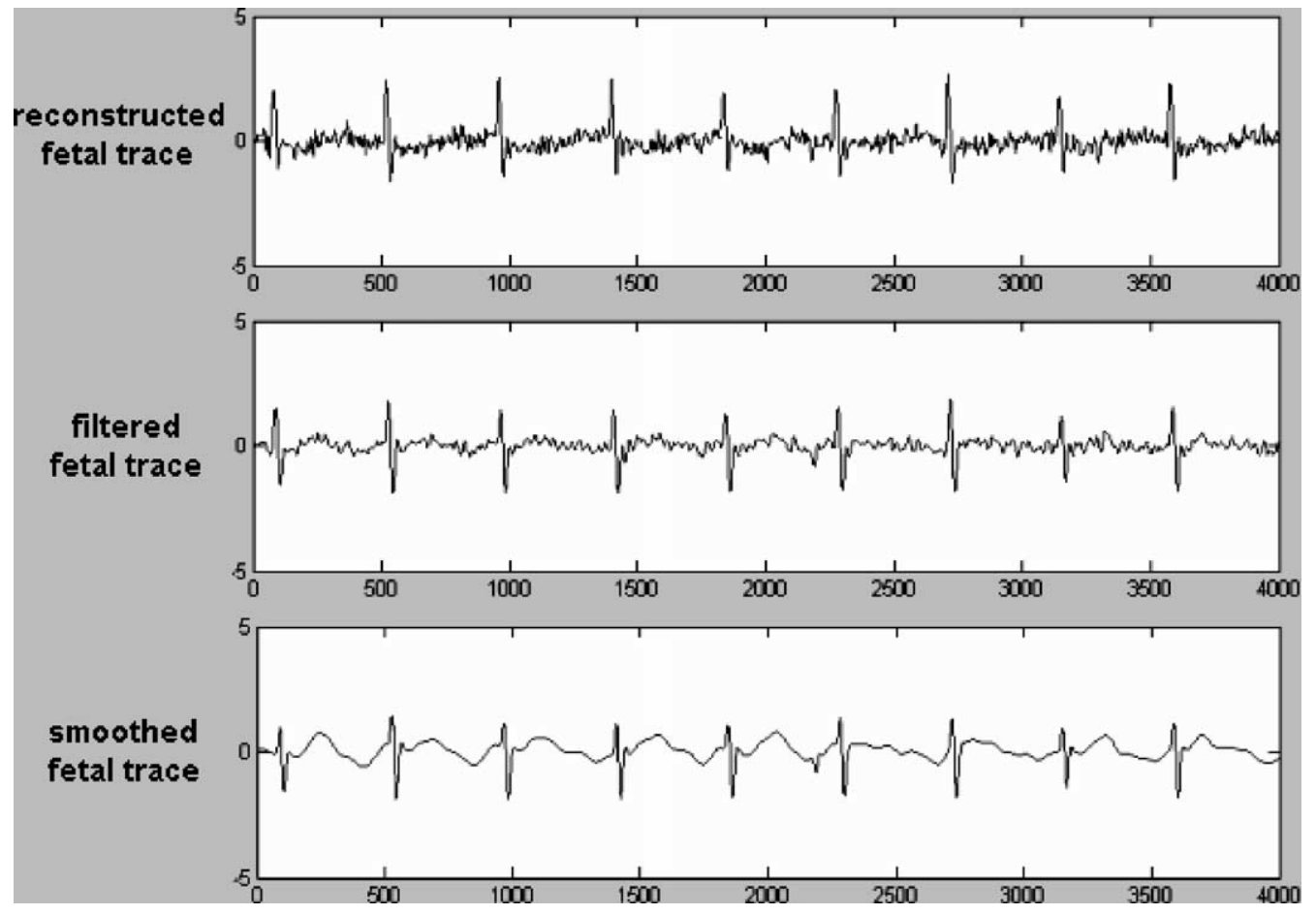

Fig. 11 Example of a fetal signal reconstructed with FastICA (upper trace), which is filtered for high frequencies (middle trace) and further smoothed (lower trace). The correct amplitude (pT) and time (ms) scales have been restored.

FastICA succeeded in the fetal signal extraction, while the other technique was ineffective.

The various rates of success, calculated for all data sets grouped in gestational periods of 4 weeks each, are summarized in Table 1. Fifty-one fetal traces could be reconstructed from the 61 available MCG recording sets (see RRS); all reconstructed fe- tal traces could be used to estimate an average FHR and its variability. Forty-four signals, out of the 51 fetal traces, had a morphology good enough to allow cardiac analysis at the level of single cardiac waves (see URS). Therefore, 44 traces, out of the 61 recorded data sets, were utilizable for extensive clinical applications (see ORS). The values reported 
Table 1 Rates of success of the FastICA algorithm in the extraction of fetal signal given in percentage; percentages are calculated in function of the gestational period

\begin{tabular}{llllll}
\hline \multicolumn{5}{c}{ Gestational period in weeks } \\
\cline { 2 - 6 } & 22nd to 37th & 22nd to 25th & 26th to 29th & 30th to 33rd & 34th to 37th \\
\hline RRS $^{\text {a }}$ & 84 & 63 & 81 & 93 & 100 \\
URS $^{\text {b }}$ & 86 & 80 & 77 & 85 & 100 \\
ORS $^{c}$ & 72 & 50 & 63 & 79 & 100 \\
\hline
\end{tabular}

${ }^{a}$ RRS stands for reconstructed rate of success.

b URS stands for useful rate of success.

' ORS stands for overall rate of success; for the detailed definitions of the rates of success refer to Section 2.7.
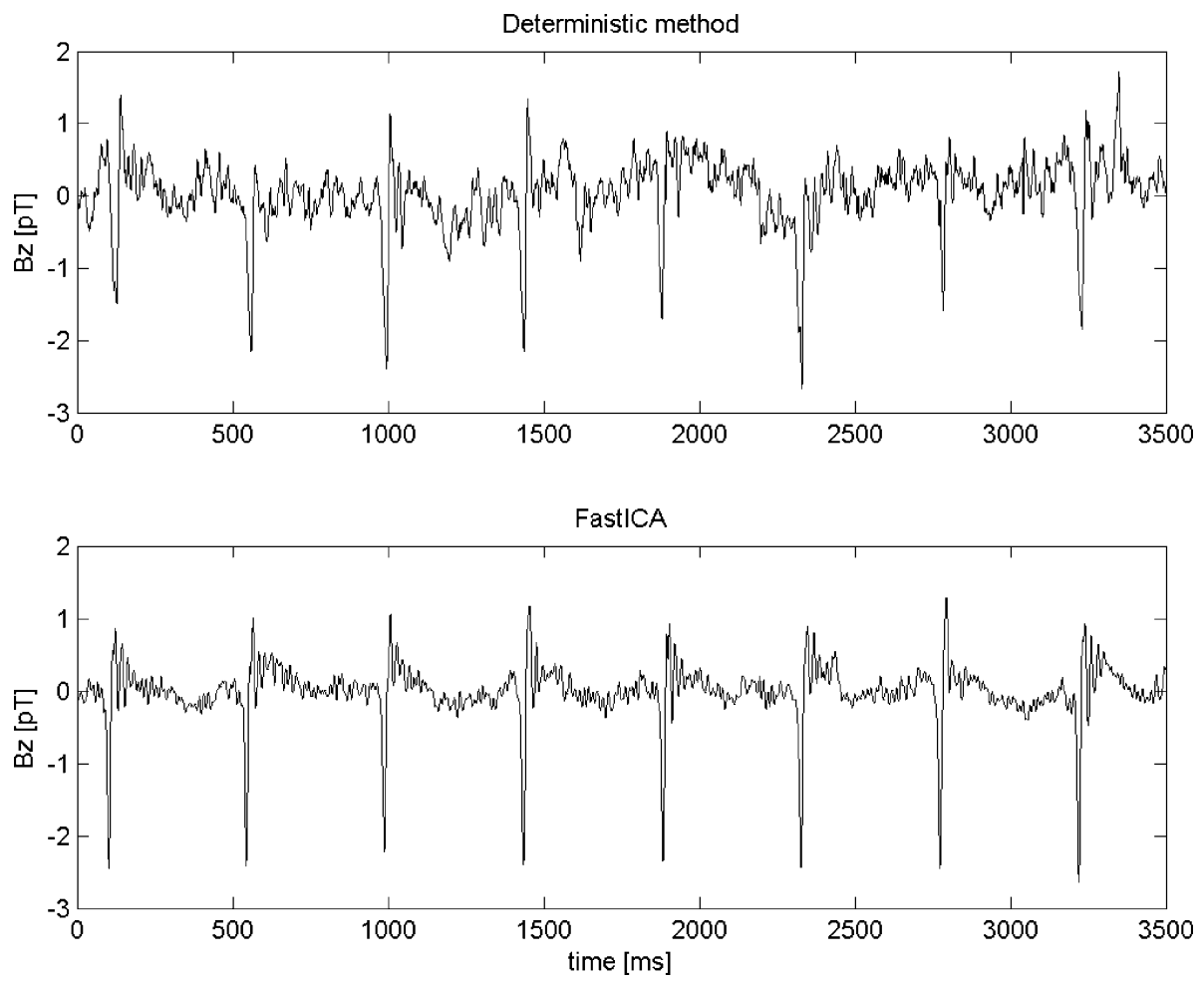

Fig. 12 Comparison between fetal signals reconstructed with the deterministic method of the maternal average beat subtraction (upper trace) and with FastICA (lower trace). The correct amplitude (pT) and time (ms) scales have been restored.

in the literature range between $80 \%$ and $85 \%$ for averaged fetal beats [41,42]; it should be underlined that in our study we reconstructed the entire time course of the signals [40].

As regards trace quality, it was estimated by comparing the FastICA results with the outcomes of the deterministic technique. This comparison showed that the signal waveforms were similar, as well as the peak-to-peak amplitudes and RR periodicities. In Fig. 12 the fetal traces obtained from the same data set using both FastICA and the mentioned method are compared. The data refer to a pregnancy at the 35th gestational week.
An inter-individual variability in the overall effectiveness of FastICA was observed. This means that not all data sets, although recorded with the same system and the same protocol, allowed for the same performance of FastICA.

We can finally mention that the amount of memory required to perform the described procedure is about $90 \mathrm{MB}$. On the other hand, the overall time necessary to obtain $N_{0}$ components is a function of values chosen for $N_{1}$ and $N_{0}$; we calculated that FastICA required, on average, $20 \mathrm{~s}$ to extract six components out of 22 input recordings of $1 \mathrm{~min}$ registration. 


\section{Discussion}

The retrieval of information on the electrical activity of the fetal heart is essential for an early and reliable diagnosis of several cardiac diseases and for an appropriate treatment planning [16-19]. The availability of such information during the last period of pregnancy is even more important, but techniques such as the CTG or the fECG have shown to be inadequate [16-19]. The development of new methods able to meet the mentioned requirements is therefore welcome, and FMCG has demonstrated to be particularly useful for the monitoring of fetal cardiac activity during the last gestational period $[22,26,39]$.

Methods working in the time domain have shown to be successful in reconstructing a fetal signal from fMCG recordings [17,23-26]. Such procedures usually involve the subtraction of an average maternal beat from the fMCG recordings; as a consequence, the fetal beats that, due to the different heart rates of the fetus and the mother, are simultaneous with the more intense maternal beats are disregarded in the reconstructed fetal trace simply because they remain hidden. Although the major features of a cardiac cycle could still be retrieved, the variability estimate of interesting cardiac intervals would be affected by an unpredictable error. An appropriate method for the accurate retrieval of the entire fetal information included in fMCG recordings is therefore necessary.

Our results prove that a procedure based on the ICA approach can be successfully applied to fMCG recordings in order to separate the fetal cardiac signal from the maternal one. The rates of success summarized in Table 1 are within the ranges given by other authors $[41,42]$ but it is worth underlining that our figures refer to the reconstruction of the time course of the cardio-electrical events occurring during the entire recording period, and not only to the estimate of an averaged beat. This means, for instance, that the fetal traces reconstructed with FastICA allow not only inter-individual studies, but also intra-individual variability studies of various important electrophysiological indicators [40].

The inter-individual variability observed in the effectiveness of FastICA may be ascribed to several factors, which affect other deterministic procedures as well. The most common reasons for a failure in the reconstruction of a reliable fetal signal are a low signal-to-noise ratio in the recorded data, uncontrolled fetal movements during the recording, maternal obesity inducing an excessive distance between the fetal heart and the magnetic sensors, unfavourable orientation of the fetal heart during data acquisition. The situation is even worse for earlier gestational epochs, characterized by weaker fetal signals. This inter-individual variability could not be removed, because it is inherently related to the type of analyzed data, and not to the reconstruction procedure.

Although the same factors that can prevent a fetal signal reconstruction from being successful may also affect the quality of the reconstructed traces, a signal waveform suitable for the recognition of fetal ventricular depolarisation and consequently for an estimate of the FHR was provided in the $84 \%$ of cases (see Table 1). In better cases also ventricular repolarization and atrial depolarisation could be estimated [40], and this regarded the $72 \%$ of the total analysed data sets (see Table 1).

Moreover, the comparison with the results of another reconstruction technique, when available, allowed verifying the reliability of the signals in terms of waveform, intensity and main periodicity, as shown in the example given in Fig. 12.

The stable output reached with the use of a large input cluster and six output components also guaranteed the reliability of the fetal signals obtained by recombining the fetal components, as determined on the basis of the periodicity, which corresponds to the heart rate, maternal or fetal.

A great advantage of FastICA, with respect to deterministic techniques working in the time domain, is its insensitiveness to the occasional and unpredictable overlapping of the maternal and fetal beats. As a consequence, no fetal beat was disregarded in the trace reconstruction process, and most information desirable by neonatologists, such as the RR frequency, the QRS amplitude, the QT interval and related variability values, could be obtained from these traces with great accuracy, as evident from the comparison with the results obtained by other research groups $[26,40]$.

Since the final target was the retrieval of signals useful for clinical purposes, an easy detection of the different intra-cardiac intervals, as well as the possibility of reconstructing cardiac magnetic mapping, would be preferred. As regards the first point, some trace filtering and smoothing could be performed to advance trace quality without altering its information content. As regards the second point, a reduced cardiac mapping was possible, in some cases, by rotating the large clusters used to reconstruct the fetal signals [43].

\section{Conclusions}

In the light of the above considerations, we can conclude that the ICA method, and in particular the FastICA algorithm, showed to be suitable for the 
reconstruction of cardiac fetal traces that might be used not only for research but also for clinical purposes. Given the weakness of the signals originating from the fetal heart, a further improvement of this technique would be desirable in order to get closer to the requirements for methods routinely used in a clinical setting. A possibility could be the use of a priori information on cardiac signal waveform. Studies have already been performed on the extraction of specific signals with temporal structure [44], and the structure of a heartbeat could be parameterized and used to advance the performances of FastICA, in terms of signal quality, rate of success and total processing time, hence permitting to extensively validate this technique in the perspective of its clinical use.

\section{Availability}

An evaluation, limited version of the software is available for academic use only; it can be required via e-mail directly from the authors.

\section{References}

[1] S. Amari, A. Cichocki, H.H. Yang, A new learning algorithm for blind signal separation, in:G. Tesauro, D.S. Touretzky, T.K. Leen (Eds.), Advances in Neural Information Processing Systems, vol. 8, The MIT Press, Cambridge MA, 1996, pp. 757-763.

[2] T. Ristaniemi, K. Raju, J. Karhunen, Jammer Mitigation in DS-CDMA Using Independent Component Analysis, in: Proc. of the IEEE Int. Conf. on Communications, New York, USA, 2002, pp. 1-5.

[3] J. Karhunen, A. Hyvärinen, R. Vigario, J. Hurri, E. Oja, Applications of neural blind separation to signal and image processing, in: Proceedings IEEE Int. Conf. on Acoustics, Speech and Signal Processing (ICASSP'97), Munich, Germany, 1997, pp. 131-134.

[4] S. Makeig, A.J. Bell, T.P. Jung, T.J. Sejnowski, Independent component analysis of electroencephalographic data, in: Advances in Neural Information Processing Systems, vol. 8, The MIT Press, Cambridge MA, 1996, pp. 145-151.

[5] A.J. Bell, T.J. Sejnowski, Learning higher-order structure of a natural sound, Network 7 (1996) 261-266.

[6] P. Comon, Independent component analysis-a new concept? Signal Process. 36 (1994) 287-314.

[7] A.J. Bell, T.J. Sejnowski, Fast blind separation based on information theory, in: Proceedings of the International Symposium on Nonlinear Theory and Applications, vol. 1, Las Vegas, Nevada, USA, 1995, pp. 43-47, pp. 43-47.

[8] V. Capdevielle, Ch. Serviere, J. Lacoume, Blind separation of wide-band sources in the frequency domain, in: Proceedings IEEE Int. Conf. on Acoustics, Speech and Signal Processing (ICASSP'95), vol. 3, Detroit, Michigan, USA, 1995, pp. 2080-2083.

[9] R. Vigário, V. Jousmäki, M. Hämäläinen, R. Hari, E. Oja, Independent component analysis for identification of artifacts in magnetoencephalographic recordings, in: Advances in Neural Information Processing, vol. 10, The MIT Press, Cambridge MA, 1998, pp. 229-235.

[10] S.S. Yoo, B. Gil Choi, J.Y. Han, H. Hee Kim, Independent component analysis for the examination of dynamic contrast-enhanced breast magnetic resonance imaging data: preliminary study, Invest. Radiol. 37 (2002) 647654.

[11] C.G. Thomas, R.A. Harshman, R.S. Menon, Noise reduction in BOLD-based fMRI using component analysis, Neuroimage 17 (2002) 1521-1537.

[12] K. Kobayashi, T. Akiyama, T. Nakahori, H. Yoshinaga, J. Gotman, Systematic source estimation of spikes by a combination of independent component analysis and RAP-MUSIC. I: Principles and simulation study, Clin. Neurophysiol. 113 (2002) 713-724.

[13] G. Kobayashi Wubbeler, A. Ziehe, B.M. Mackert, K.R. Muller, L. Trahms, G. Curio, Independent component analysis of noninvasively recorded cortical magnetic DC-fields in humans, IEEE Trans. Biomed. Eng. 47 (2000) 594-599.

[14] F. Esposito, E. Formisano, E. Seifritz, R. Goebel, R. Morrone, G. Tedeschi, F. Di Salle, Spatial independent component analysis of functional MRI time-series: to what extent do results depend on the algorithm used? Hum. Brain Mapp. 16 (2002) 146-157.

[15] G. Stroink, W. Moshage, S. Achenbach, Cardiomagnetism, in: W. Andrä and H. Nowak (Eds.), Magnetism in Medicine, Wiley-VCH, Berlin, 1998, pp. 136-189.

[16] C.S. Kleinman, R.L. Donnerstein, C.C. Jaffe, G. Devore, E.M. Weinstein, D. Lynch, N.S. Talner, J.C. Hobbins, R.L. Berkowitz, Fetal echocardiography. A tool for evaluation of in utero cardiac arrhythmias and monitoring of in utero therapy: Analysis of 71 patients, Am. J Cardiol. 51 (1983) 237-243.

[17] P. van Leeuwen, B. Hailer, W. Bader, J. Geissler, E. Trowitzsch, D.H. Gronemeyer, Magnetocardiography in the diagnosis of fetal arrhythmia, Br. J. Obstet. Gynaecol. 106 (1999) 1200-1208.

[18] M. Peters, J. Crowe, J.F. Piéri, H. Quartero, B. Hayes-Gill, D. James, J. Stinstra, S. Shakespeare, Monitoring the fetal heart non-invasively: a review of methods, J. Perinat. Med. 29 (2001) 408-416.

[19] E. Namburg, T. Riesenfeld, O. Axelsson, Fetal tachycardia: intrauterine and postnatal course, Fetal Diagn. Ther. 12 (1997) 205-209.

[20] T. Menendez, S. Achenbach, E. Beinder, M. Hofbeck, L. Klinghammer, H. Singer, W. Moshage, W.G. Daniel, Usefulness of magnetocardiography for the investigation of fetal arrhythmias, Am. J. Cardiol. 88 (2001) 334-336.

[21] R.T. Wakai, J.F. Strasburger, Z. Li, B.J. Deal, N.L. Gotteiner, Magnetocardiographic rhythm patterns at initiation and termination of fetal supraventricular tachycardia, Circulation 107 (2003) 307-312.

[22] J.G. Stinstra, M.J. Peters, The influence of fetoabdominal tissues on fetal ECGs and MCGs, Arch. Physiol. Biochem. 110 (2002) 165-176.

[23] T. Menendez, S. Achenbach, E. Beinder, M. Hofbeck, O. Schmid, H. Singer, W. Mosage, Prenatal diagnosis of QT prolongation by Magnetocardiography, Pacing Clinical Electrophysiol. 23 (2000) 1305-1307.

[24] R.T. Wakai, A. C: Leuthold, L. Cripe, C.B. Martin, Assessment of fetal rhythm in complete congenital heart block by magnetocardiography, Pacing Clin. Electrophysiol. 23 (2000) 1047-1050.

[25] H. Horigome, J. Shiono, S. Shigemitsu, M. Asaka, A. Matsui, A. Kandori, T. Miyashita, Detection of cardiac hypertrophy in the fetus by approximation of the current dipole using magnetocardiography, Pediatr. Res. 50 (2001) 242-245. 
[26] J.G. Stinstra, E. Golbach, P. van Leeuwen, S. Lange, T. Menendez, W. Moshage, E. Schleussner, C. Kaehler, H. Horigome, S. Shigemitsu, M.J. Peters, Multicentre study of fetal cardiac time intervals using magnetocardiography, Br. J. Obstet. Gynaecol. 109 (2002) 1235-1243.

[27] Z. Dunajski, M.J. Peters, Development of the fetal magnetocardiogram from the 13th week of gestation onward, in: C. Baumgartner et al. (Eds.), Biomagnetism: Fundamental Research and Clinical Applications, Elsevier Science, IOS Press, Amsterdam, 1995, pp. 704-708.

[28] A. Pasquarelli, S. Di Luzio, Instrumentation and methods for biomagnetic measurement, Phys. Med. 9 (1993) 249266.

[29] H.P. Muller, P. Godde, K. Czerski, R. Agrawal, G. Feilcke, K. Reither, K.J. Wolf, M. Oeff, Localization of a ventricular tachycardia-focus with multichannel magnetocardiography and three-dimensional current density reconstruction, J. Med. Eng. Technol. 23 (1999) 108-115.

[30] S. Comani, B. Merlino, S. Di Luzio, S.N. Ernè, G.L. Romani, Magnetic map analysis during ventricular repolarization to differentiate between normal subject and patients affected by cardiac hypertrophy, Physica Medica 17 (2001) 9-15.

[31] S. Comani, A. Tartaro, A. Lagatta, G. Morana, S. Di Luzio, G.L. Romani, Magnetocardiographic functional imaging and integration with 3D MRI reconstruction of the heart: preliminary results for source localization during myocardium activation, Physica Medica 19 (2003) 119-130.

[32] A. Lagatta, S. Comani, M. Stefanachi, S. Di Luzio, Can a Virtual Extension of a Planar Multi-channel MCG System improve the Localization of Sites of the Cardiac Electrical Activity?, Biomedizinische Technik 48 (2004) in press.

[33] S. Della Penna, C. Del Gratta, C. Granata, A. Pasquarelli, V. Pizzella, R. Rossi, M. Russo, K. Torquati, S.N. Ernè, Biomagnetic systems for clinical use, Philos. Mag. B 80 (2000) 937-948.

[34] A. Bell, T. Sejnovsky, An information-maximization approach to blind separation and blind deconvolution, Neural Comput. 7 (1995) 1129-1159.
[35] J.F. Cardoso, Iterative techniques for blind source separation using only fourth-order cumulants, in: Proceedings EUSIPCO, Brussels, Belgium, 1992, pp. 739-742.

[36] A. Hyvärinen, E. Oja, A fast fixed point algorithm for independent component analysis, Neural Comput. 9 (1997) 283-292.

[37] The FastICA MATLAB package, http://www.cis.hut.fi/ projects/ica/fastica/ (1998).

[38] A. Hyvärinen, Fast and robust fixed-point algorithms for independent component analysis, IEEE Trans. Neural Networks 10 (1999) 626-634.

[39] J.G. Stinstra, M.J. Peters, H.W.P. Quartero, Extracting reliable data from the fetal MCG, in: Proceedings of the International Conference on Biomagnetism, Helsinki, Finland, 2000, pp. 591-594.

[40] S. Comani, M. Liberati, E. Gabriele, A. Santarelli, A. Lagatta, D. Mantini, M. Stefanachi, G. Cancellieri, S. Di Luzio, G.L. Romani, Fetal Intra-cardiac Intervals for Different Gestational Epochs as Evaluated from Fetal Magnetocardiograms, Biomedizinische Technik 48 (2004) in press.

[41] H. Horigome, M.I. Takahashi, M. Asaka, S. Shigemitsu, A. Kandori, K. Tsukada, Magnetocardiographic determination of the development changes in $\mathrm{PQ}, \mathrm{QRS}$ and $\mathrm{QT}$ intervals in the foetus, Acta Pediatr. 89 (2000) 64-67.

[42] C. Kaler, E. Schleussner, B. Grimm, A. Schneider, U. Schneider, H. Nowak, H.J. Seewald, Fetal magnetocardiography: development of the fetal cardiac time intervals, Prenatal Diag. 22 (2002) 408-414.

[43] D. Mantini, S. Comani, P. Pennesi, G. Cancellieri, Fetal Tailoring of the Independent Component Analysis to multi-channel fMCG recordings for an optimal reconstruction of the fetal cardiac signal, Biomedizinische Technik $48(2004)$ in press.

[44] A.K. Barros, A. Chichocki, Extraction of specific signals with temporal structure, Neural Computat. 13 (2001) 195203. 